\title{
Validación de un Prototipo Electrónico para el Control de Fertirrigación e Invernaderos ${ }^{1}$
}

\section{Validation of an electronic prototype for fertigation and greenhouse control.}

\author{
Y. Valencia, C. A. Henao y M. F. Polanco \\ Recibido: 26 de junio de 2020 - Aceptado: 26 de junio de 2021
}

\begin{abstract}
Resumen-En este artículo se presenta el procedimiento de validación de un prototipo electrónico de fertirrigación y control ambiental en un invernadero. La metodología compara la información recopilada en sitio, confrontado el efecto de las acciones de control sobre las variables fisicoquímicas del suelo cercanas a la raíz de la planta y la de su ambiente circundante. La información recopilada en el tiempo es analizada para identificar y evaluar el desempeño del hardware, así como la funcionalidad del controlador. Los resultados permiten evidenciar el aporte de método introducido, dado que se logra validar el prototipo en cada una de las etapas del proceso de control, manteniendo los valores de las variables evaluadas, cercanos al valor de referencia. Finalmente, se logra controlar todas las variables con un único controlador, y se destaca la importancia de contar con interfaz de usuario en sitio y a través de la red para la gestión de controlador. Palabras clave- Control
\end{abstract}

${ }^{1}$ Producto derivado del proyecto de investigación "Desarrollo de un Sistema Controlado de Fertirrigación y Control Ambiental”, apoyado por la Universidad Nacional Abierta y a Distancia UNAD.a través de la Escuela de Ciencias Agrícolas, Pecuarias y del Medio Ambiente ECAPMA

Y. Valencia, Universidad Nacional Abierta y a Distancia UNAD, Dosquebradas, Colombia, email: yenier.valencia@unad.edu.co.

C. A. Henao, Línea de Electrónica y Telecomunicaciones Tecnoparque, Pereira, Colombia, email: c_henao_86@hotmail.com

M. F. Polanco, Universidad Nacional Abierta y a Distancia UNAD, Dosquebradas, Colombia, email: manuel.polanco@unad.edu.co.

Como citar este artículo: Valencia, Y., Polanco, M. F., y Henao, C. A. Validación de un Prototipo Electrónico para el Control de Fertirrigación e Invernaderos, Entre Ciencia e Ingeniería, vol. 15, no. 29, pp. 28-36, enerojunio 2021. DOI: https://doi.org/ 10.31908/19098367.1705.

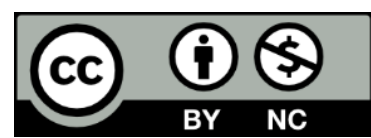

Attribution-NonCommercial 4.0 Intenational (CC By-NC 4.0) automático, Fertirriego, Interfaz de usuario, Internet de las cosas, Invernadero, Riego por goteo, Sensor.

Abstract-This article presents the validation procedure of an electronic prototype of fertigation and environmental control in a greenhouse. The methodology compares the information collected on site, comparing the effect of control actions on the physical-chemical variables of the soil near the root of the plant and that of its surrounding environment. The information collected over time is analyzed to identify and evaluate the performance of the hardware, as well as the functionality of the controller. The results show the contribution of the introduced method, since it is possible to validate the prototype in each of the stages of the control process, keeping the values of the evaluated variables close to the reference value. Finally, it is possible to control all the variables with a single controller, and the importance of having a user interface on site and through the network for controller management.

Keywords - Automatic control, Fertigation, User interface, Internet of things, Greenhouse, Drip irrigation, Sensor.

\section{DEFINICIONES Y NOMENCLATURA}

$\begin{array}{cl}r(t) & \text { Señal de referencia del sistema. } \\ r n(t) & \text { Señal de referencia interfaz de usuario HMI (a). } \\ r m(t) & \text { Señal de referencia interfaz de usuario HMI (b). } \\ h(t) & \begin{array}{l}\text { Señal de realimentación del sistema total. } \\ \text { Señal de realimentación sensor de temperatura del }\end{array} \\ T e m(t) & \begin{array}{l}\text { aire }{ }^{\circ} \mathrm{C} . \\ \text { Señal de realimentación de humedad relativa del }\end{array} \\ R H(t) & \begin{array}{l}\text { aire. } \\ \text { Señal de realimentación tensión del agua en el suelo }\end{array} \\ T(t) & \begin{array}{l}\text { en pa. } \\ p H(t)\end{array} \\ \text { Señal realimentación acidez del suelo. } \\ C E(t) & \begin{array}{l}\text { Señal de realimentación Conductividad Eléctrica CE } \\ \text { del suelo S/m. }\end{array} \\ H(t) & \text { Señal de realimentación tensión en el suelo en pa. }\end{array}$




$$
\begin{array}{cl}
u(t) & \text { Señal de control del sistema. } \\
y(t) & \text { Visualización de los valores de } r(t) \text { y } h(t) . \\
y n(t) & \text { Visualización de los valores } r(t) \text { y } h(t) \text { en HMI } \\
& \text { (a). } \\
y m(t) & \begin{array}{l}
\text { Visualización de los valores } r(t) \text { y } h(t) \text { en HMI } \\
\text { (b). } \\
\text { Señal de realimentación de humedad relativa del aire } \\
\text { en porcentaje. }
\end{array}
\end{array}
$$

\section{II.INTRODUCCIÓN}

$\mathrm{L}$ A fertirrigación y el cultivo bajo invernadero, son técnicas que permite gestionar el riego, fertilización, y control de variables ambientales en un cultivo. La modernización en los sistemas de riego y control ambiental de los invernaderos, han permito pasar de una operación manual a procesos automáticos donde la dependencia de personal es mínima. La automatización de estas ofrece múltiples ventajas, en primer lugar, mejora la distribución del agua y nutrientes en el suelo con una eficiencia de riego entre 90 y $95 \%$ [1], en segundo lugar, el cultivo bajo invernadero permite aumentar el rendimiento por hectárea de algunos cultivos [2], finalmente, cabe destacar la posibilidad de liberar parte de la mano de obra y minimizar errores del personal en el proceso.

Durante las últimas décadas, se han diseñado e implementado múltiples sistemas de control automático de riego, fertilización y clima en invernaderos, tal es el caso de [3], donde se plantean herramientas modernas para la gestión del riego de precisión mediante la estimación de evapotranspiración y el coeficiente del cultivo, y al igual que en esta validación, mediante el control continuo de humedad en la zona radicular de la planta. En [4] se propone el diseño de un controlador automático de Fertirrigación, y en [5] la automatización de condiciones climáticas bajo invernadero. Ahora bien, si el conjunto de estos desarrollos facilita las tareas de Fertirrigación y control ambiental, no son una solución integral que permita realizar múltiples tareas. Otros diseños incorporan múltiples funciones, como en el caso de [6], [7] donde se plantea el control automático de clima y Fertirrigación; sin embargo, estos incorporan funcionalidades muy específicas y no cuentan con una interfaz de usuario programable que permita ajustar los requerimientos del cultivo. Otros controladores están orientados desde aplicaciones móviles [8], desarrollo de software [9] o interfaz física [10]; sin embargo, estos no cuentan con múltiples plataformas que permita la administración Offline y Online de los dispositivos. La mayor parte de las pruebas documentales están orientadas al diseño de los desarrollos tecnológicos [4], [11], simulaciones y/o validación en maquetas a escala [9]; sin embargo, no permiten establecer claramente el desempeño de los desarrollos en un entorno real. Es por esto que, en este artículo, se documenta el proceso metodológico que permitió validar un prototipo destinado para el control de riego. El método permite poner a prueba el hardware electrónico, así como la robustez del diseño, permitiendo evaluar el consumo energético, rango para la transferencia y recepción de información del controlador, también se determina la operatividad de acuerdo con valores de referencia del equipo. Los experimentos se realizaron por una ventana de tiempo de un mes, la información del cultivo fue recopilada mediante el almacenamiento de datos Online y Offline. Los resultados justipreciaron el desempeño del prototipo en el tiempo, determinando las dinámicas de las acciones de control dadas los valores de referencia de las variables ambientales, así como los datos recopilados por los instrumentos de medición.

El artículo se organiza de la siguiente manera, en la parte dos se enseña la metodología de trabajo, en la parte tres se muestran los resultados obtenidos, por último, las conclusión y discusión de la experiencia.

\section{Metodología}

La aplicación del dispositivo electrónico se fundamenta en el monitoreo y control en tiempo real de las variables que tienen incidencia en el desarrollo de un cultivo, de esta forma, determinar la necesidad hídrica, nutricional y ambiental del cultivo para suplir los requerimientos en el momento justo y cantidades precisas. En esta sección se define los elementos técnicos del controlador, sensores, actuadores, comunicación e interfaz de usuario. Además, se enseña la estrategia de validación de funcionamiento del sistema.

La Fig. 1 muestra la estructura general del controlador a través de un diagrama de bloques, este resume los elementos que lo conforman, por ejemplo, la interfaz de usuario in situ HMI (a) y remota HMI (b), Sensores, Elementos de control, y métodos de Comunicación inalámbrica, serie y paralela utilizados en los diferentes periféricos de entrada y salida del proceso y el microcontrolador $\mu \mathrm{C}$. Adicional en la Fig. 2 se muestra el prototipo de controlador ensamblado con los elementos principales como el $\mu \mathrm{C}$, potencia, HMI (a) y módulo de comunicaciones dado en la Fig. 1.

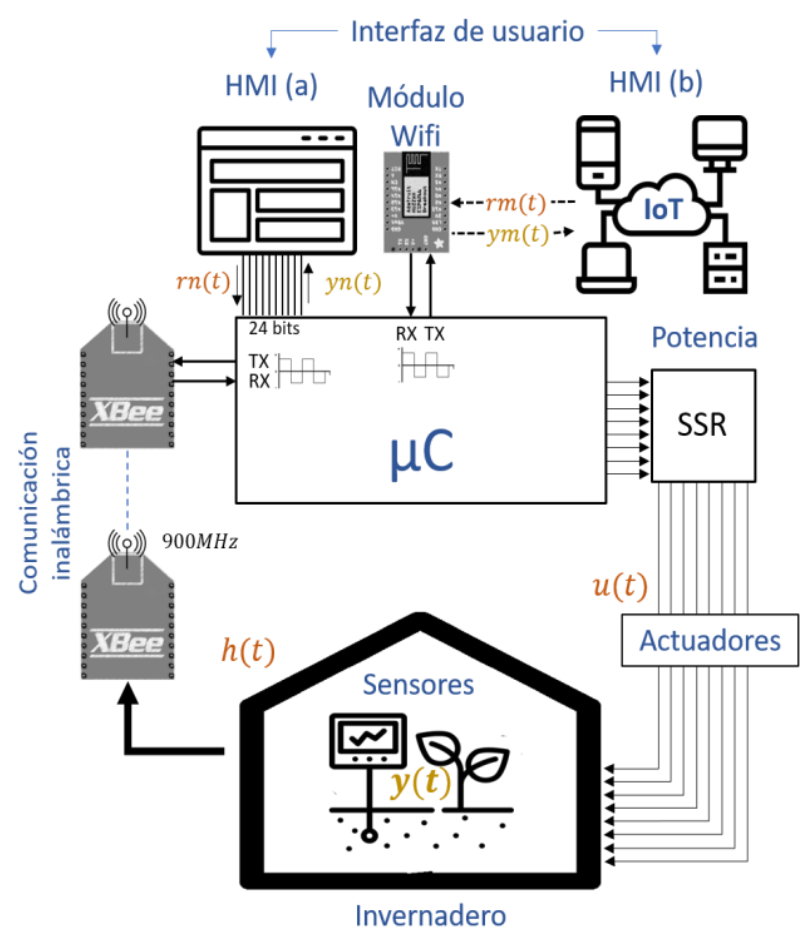

Fig. 1. Diagrama de bloques del proceso. Fuente: Autores 


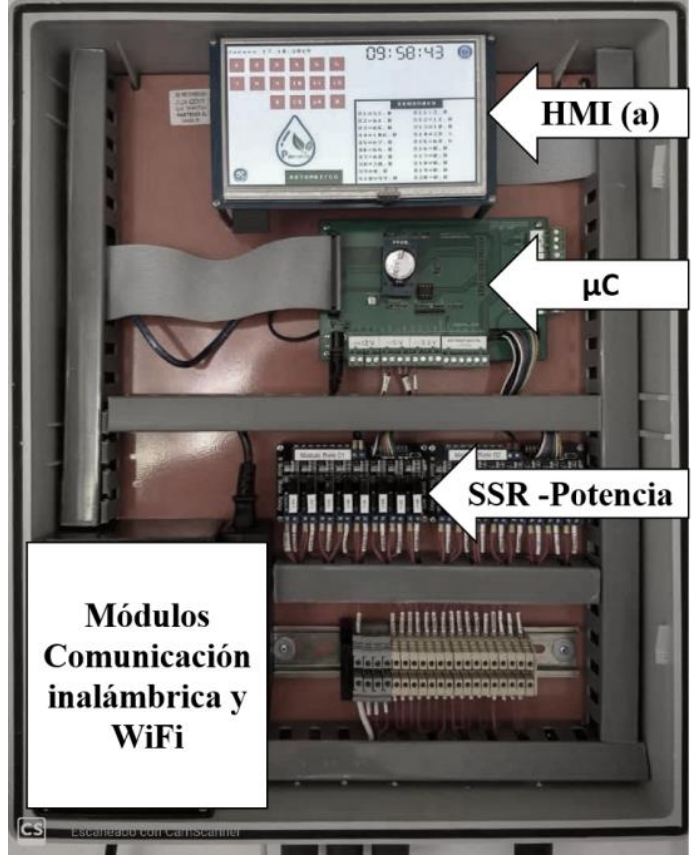

Fig. 2. Imagen del prototipo controlador

En el sistema, la señal de referencia de la ecuación (1) es determinada por los parámetros de configuración de cada salida de control (véase Tabla I) que se establecen a través de las interfaces de usuario HMI (a) y HMI (b).

$$
r(t)=[r n(t), r m(t)]
$$

Por otro lado, la señal de realimentación determinada por $h(t)=[\operatorname{Tem}(t), R H(t), H(t), p H(t), C E(t)]$ está asociada a los sensores instalados en el invernadero. Por otro lado, la acción de control $u(t)$ es aplicada a los actuadores del sistema para preservar los valores de las variables ambientales dentro de un rango de referencia deseado, a través de ventiladores y/o extractores para el control de las señales $T e m(t)$ y $R H(t)$, similarmente electroválvulas y motobombas para el control de $H(t)$, finalmente inyectores Venturi para el control de $p H(t)$ y $\quad C E(t)$. Notar entonces que $u(t)=\left[u_{1}(t), u_{2}(t), \ldots \ldots, u_{n}(t)\right]$ donde $u_{n}(t)$ corresponde a la señal de control del $n$ - ésimo actuador del sistema.

Por último, la salida del sistema de control $y(t)$ corresponde a la visualización de los valores del término $h(t)$, parámetros de configuración $r(t)$ y estado de las salidas (activa ON-inactiva OFF) de control $u(t)$. Es de notar que la respuesta $y(t)$ puede escribirse tomando la señal $y n(t)$ concatenando con $y m(t)$, de tal manera que $y(t)=[y n(t), y m(t)]$.

\section{A. Interfaz de usuario}

El sistema cuenta con dos interfaces de usuario, consiguiendo a través de estas configurar los parámetros $r(t)$ enseñados en la Tabla I; simultáneamente se visualiza en tiempo real los valores de la señal $h(t)$ y el estado de las acciones de control $u(t)$. De acuerdo con los parámetros $r(t)$ vinculados en la interfaz de usuario, el sistema establece las condiciones de control para cada una de las salidas del controlador.

TABLA I

PARÁMETROS DE REFERENCIA

\begin{tabular}{|l|c|l|}
\hline \multicolumn{1}{|c|}{ Parámetro } & Rango & \multicolumn{1}{c|}{ Descripción } \\
\hline $\begin{array}{l}\text { Hora de encendido } \\
\text { Hora de apagado }\end{array}$ & $00: 00-23: 59$ & $\begin{array}{l}\text { Establece el rango de tiempo } \\
\text { de control automático. }\end{array}$ \\
\hline $\begin{array}{l}\text { Tiempo de activación } \\
\text { Tiempo de no activación }\end{array}$ & $>0$ (Min o Seg) & $\begin{array}{l}\text { Define el intervalo de } \\
\text { operación permitido de una } \\
\text { variable }\end{array}$ \\
\hline $\begin{array}{l}\text { Mínimo de la variable } \\
\text { Máximo de la variable }\end{array}$ & $>0 \&<99,999$ & $\begin{array}{l}\text { Estable el rango deseado de } \\
\text { una variable }\end{array}$ \\
\hline $\begin{array}{l}\text { Sensor asociado a la } \\
\text { salida } \\
\text { Sensor de pH } \\
\text { Sensor de CE }\end{array}$ & $1-20$ & $\begin{array}{l}\text { Selecciona el ID del sensor } \\
\text { asociado a cada una de las } \\
\text { variables ambientales }\end{array}$ \\
\hline Valor pH deseado & $1-14$ & $\begin{array}{l}\text { Define el valor deseado de } \\
\text { pH }\end{array}$ \\
\hline Valor CE deseado & $0-30 \mathrm{mS} / \mathrm{cm}$ & $\begin{array}{l}\text { Define el valor deseado de } \\
\text { CE }\end{array}$ \\
\hline $\begin{array}{l}\text { Fertirrigación } \\
\text { Motobomba } \\
\text { pH } \\
\text { CE } \\
\text { Humedad }\end{array}$ & ON-OFF & $\begin{array}{l}\text { Activa o desactiva el control } \\
\text { automático de las funciones } \\
\text { del controlador }\end{array}$ \\
\hline
\end{tabular}

La interfaz de usuario HMI (a) se soporta con un módulo de pantalla táctil a color de 7.0" con una resolución de 800x480 pixel, la cual se conecta con el controlador a través de una comunicación peraleda de 24 bits. Esta pantalla soporta la lectura y escritura de datos en una memoria MicroSD permitiendo almacenar el historial de datos del controlador $h(t)$. La HMI (a) se establece como interfaz principal, dado que no depende de una conexión internet para su operación.

Por otro lado, la interfaz de usuario HMI (b) se soporta en la plataforma de internet de las cosas IoT [12], a través de la cual, se tiene control total del dispositivo de forma remota a través de una aplicación móvil disponible en iOS y Android. Teniendo en cuenta que el controlador no es dependiente de la plataforma IoT, la interfaz de usuario HMI (b) se establece como secundaria.

\section{B. Comunicaciones}

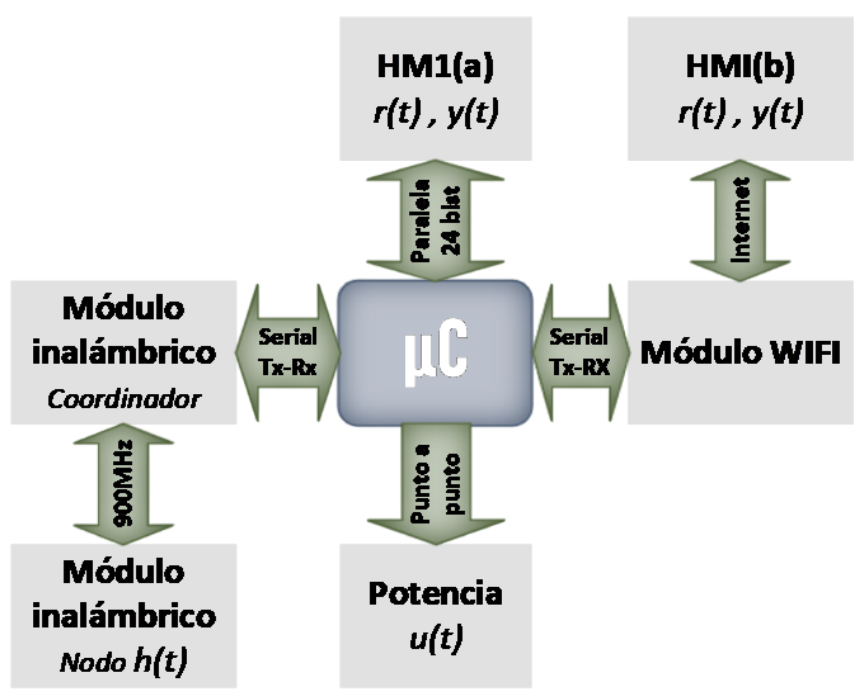

Fig. 3. Diagrama de bloques de comunicaciones. Fuente: Autores. 
Dentro de este marco, la Fig. 3 enseña la estructura de transferencia de información entre el controlador, las interfaces de usuario, sensores y elementos de potencia. El microcontrolador utilizado para la comunicación entre los diferentes periféricos de entrada y salida es el AT91SAM3X8E del módulo Arduino duo [13], que dispone de 4 puertos seriales. En esta desarrollo el puerto Serie 1 establece la comunicación con el módulo inalámbrico Coordinador que recibe los datos de las variables $h(t)$ de los sensores instalados en campo, a través del protocolo ZigBee IEEE 802.15.4 y como elementos de hardware los módulos Xbee PRO S3B [14]; Estos permiten la implementación de redes punto a multipunto, modos de bajo consumo energético, largo alcance, hasta $15.5 \mathrm{~km}$ en condiciones ideales con antena de alta ganancia a una frecuencia de $900 \mathrm{MHz}$, permitiendo la escalabilidad del sistema en proyectos con tramos de largo alcance y con múltiples nodos de medidas. Por otro lado, el puerto Serie 2 es utilizado para la comunicación bidireccional con el módulo NodeMCU [15] que incorpora el chip Wifi ESP2268 (Módulo WIFI) para entablar la comunicación con la plataforma IoT Blynk, a través de internet por medio de: APP - Servidor Blynk - N1odeMCU - Controlador. Finalmente, la comunicación paralela de 24 bits de la HMI(a) se realiza a través de un módulo de Pantalla Touch resistiva a color, la cual incorpora el controlador gráfico SSD1963 [16] y el controlador de pantalla táctil XPT2046 [17].

\section{Sensores}

Las mediciones se concentran en las variables $h(t)$ cercanas a la planta para determinar sus necesidades como se ilustra en la Fig. 4. Adicionalmente, en la Tabla II se describen las características de los sensores utilizados en la validación del prototipo.

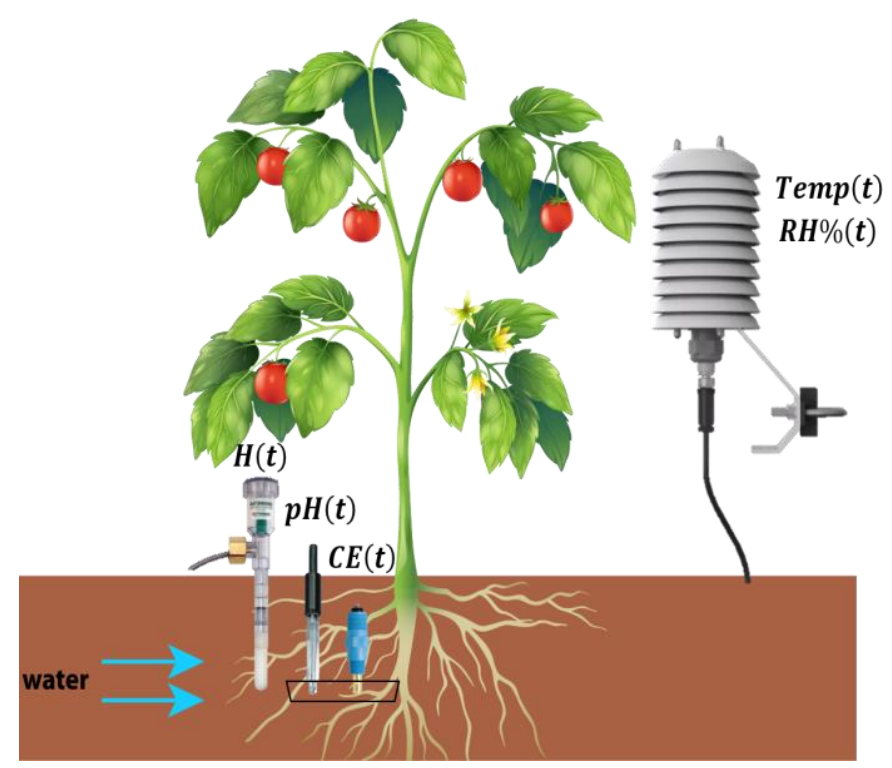

Fig. 4. Medición de variables en raíz y ambiente cercano a la planta. Fuente: Autores
TABLA II

CARACTERÍSTICA DE LOS SENSORES

\begin{tabular}{|c|c|c|}
\hline$h(t)$ & $\begin{array}{l}\text { Referencia } \\
\text { Sensor }\end{array}$ & Características \\
\hline$\frac{\operatorname{Tem}(t)}{R H(t)}$ & AM2315 [18]. & $\begin{array}{l}\text { Incorpora un microcontrolador en su } \\
\text { interior que proporciona una lectura } \\
\text { simple I2C de los valores calibrados. }\end{array}$ \\
\hline$H(t)$ & $\begin{array}{l}\text { Tensiómetro } \\
\text { Irrometer [19]. }\end{array}$ & $\begin{array}{l}\text { Rápido tiempo de respuesta, disponible } \\
\text { en diferentes longitudes y su medición } \\
\text { no se ve afectada por la temperatura y la } \\
\text { salinidad del suelo. }\end{array}$ \\
\hline$p H(t)$ & $\begin{array}{l}\text { Electrodo } \\
\text { combinado de } \\
\text { pH de vidrio HI } \\
1090 \mathrm{~B} / 5[20] .\end{array}$ & \multirow{2}{*}{$\begin{array}{l}\text { Características industriales resistentes y } \\
\text { adecuados para aplicaciones de } \\
\text { inmersión permanente en soluciones } \\
\text { contaminadas. } \\
\text { Un Transmisor análogo con salida } \\
\text { galvánica aislada bajo el estándar 4-20 } \\
\text { mA es necesario para la medición del } \\
\text { electrodo de pH y sonda de CE, este } \\
\text { incorpora compensación automática de } \\
\text { temperatura [21]. }\end{array}$} \\
\hline$C E(t)$ & $\begin{array}{l}\text { Sonda de } \\
\text { conductividad } \\
\text { de flujo } \\
\text { continuo HI } \\
3001[22] . \\
\end{array}$ & \\
\hline
\end{tabular}

\section{Actuadores}

Como actuadores del proceso, se implementa un sistema hidráulico, ventiladores y extractores dependientes de la acción de control $u(t)$. Esta se acopla mediante un circuito de potencia con relés de estado sólido SSR con el objetivo de evitar ruido eléctrico en el sistema y aislar las cargas inductivas generadas por los boninas de los contactores, motores y las electroválvulas [23]. El esquema hidráulico se puede resume en la Fig. 5, compuesto principalmente por una motobomba "M", electroválvulas e inyectores tipo Venturi.

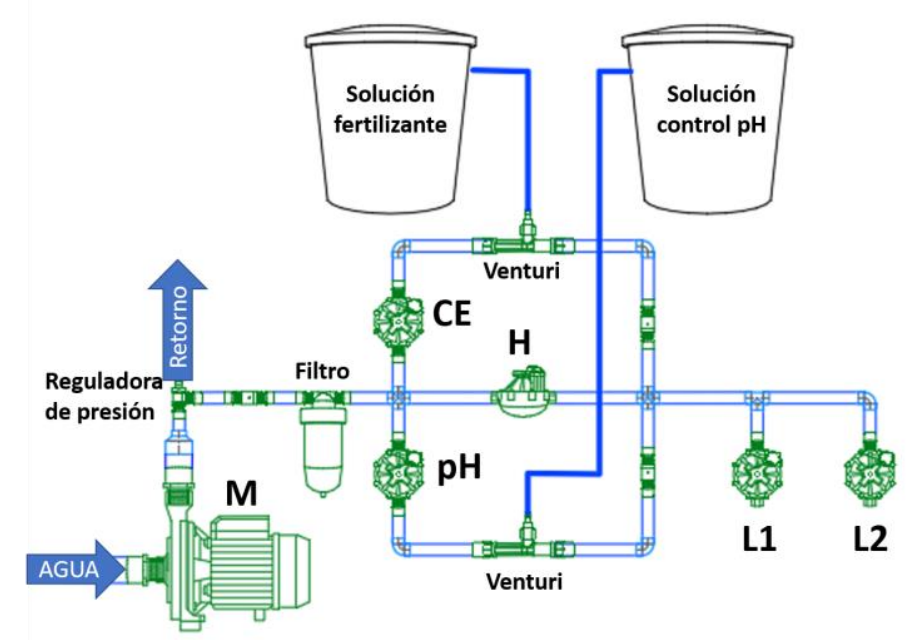

Fig. 5. Actuadores: Sistema hidráulico dependiente de la acción de control $u(t)$. Fuente: Autores

En este sentido, en la Tabla III, se describe cada una de las acciones de control $u(t)$ en el sistema hidráulico, ventiladores y extractores, así como la variable afectada $h(t)$. 
TABLA III.

\begin{tabular}{|c|c|c|c|}
\hline Id & Actuador & Acción de control $u(t)$ & $\begin{array}{c}h(t) \\
\text { afectada }\end{array}$ \\
\hline $\mathrm{CE}$ & Electroválvula & $\begin{array}{l}\text { Permite el flujo de agua a través del } \\
\text { inyector Venturi de Fertilizante }\end{array}$ & $C E(t)$ \\
\hline $\mathrm{pH}$ & Electroválvula & $\begin{array}{l}\text { Permite el flujo de agua a través del } \\
\text { inyector Venturi de la solución } \\
\text { reguladora de } \mathrm{pH}\end{array}$ & $p H(t)$ \\
\hline $\mathrm{H}$ & Electroválvula & $\begin{array}{l}\text { Electroválvula: Permite el flujo de } \\
\text { agua sin adición de fertilizante o } \\
\text { solución reguladora de } \mathrm{pH}\end{array}$ & $H(t)$ \\
\hline L1 & Electroválvula & $\begin{array}{l}\text { Electroválvula: Permite el flujo de la } \\
\text { solución hacia el sistema de riego } \\
\text { por goteo }\end{array}$ & $\begin{array}{l}C E(t) \\
p H(t) \\
H(t)\end{array}$ \\
\hline $\mathrm{L} 2$ & Electroválvula & $\begin{array}{l}\text { Electroválvula: Permite el flujo de la } \\
\text { solución hacia el sistema de } \\
\text { nebulización }\end{array}$ & $\begin{array}{l}\operatorname{Tem}(t) \\
R H(t)\end{array}$ \\
\hline V & $\begin{array}{l}\text { Ventiladores y } \\
\text { extractores }\end{array}$ & $\begin{array}{l}\text { Permite la circulación de aire en el } \\
\text { invernadero }\end{array}$ & $\begin{array}{l}\operatorname{Tem}(t) \\
R H(t)\end{array}$ \\
\hline
\end{tabular}

E. Diseño de validación

El objetivo principal de la validación es evidenciar y cuantificar el desempeño del sistema, conservando las variables del cultivo $h(t)$ de acuerdo con los parámetros de referencia $r(t)$ configurados por el usuario. Es importante aclarar que, en este artículo no se analizan los resultados del desarrollo productivo del cultivo.

La prueba de validación del sistema se realiza en el invernadero del CIAB de la UNAD, el cual tiene un área de $484 \mathrm{~m}^{2}$ y cuenta con toda la infraestructura hidráulica, eléctrica y demás actuadores necesarios para la validación del prototipo de control de Fertirrigación ambiente en un invernadero [24]. La ventana de análisis corresponde a un mes, tomando y almacenando lecturas por minuto, también se recopilan los valores y los instantes de tiempo cuando se produce cambios de estado de la señal $u(t)$. Es de aclarar que la plataforma IoT usada en este trabajo realiza un promedio por minutos de los valores de las variables medidas (esto es promedio de $h(t)$ y $u(t)$ ), en consecuencia, es imperativo calcular la media aritmética para los valores de $h(t)$ recopilados localmente así comparar contra los registrados en la IoT.

Dada la extensión del invernadero se espera que, bajo condiciones homogéneas de clima, suelo y riego por goteo autocompensado en el interior de este, el monitoreo de las variables $h(t)$ en cultivo, se realice mediante un único módulo de sensores inalámbrico instalado en una planta denominada "Planta testigo" tal como se ilustra en la Fig. 4. Es importante agregar que la validación, se realiza en un cultivo de propagación de cormos de plátano Dominico-Hartón en surcos con distancia de siembra de $80 \mathrm{~cm}$; si bien es cierto que no se evalúa el desarrollo del cultivo, se pretende ajustar los parámetros de referencia de acuerdo con los requerimientos de clima y suelo del cultivo [25], [26].

La recolección de los datos de $h(t)$ y $u(t)$ se realiza durante un mes, mediante la plataforma IoT y el almacenamiento de datos $h(t)$ a través de memoria MicroSD conectada en el $\mu \mathrm{C}$ (microcontrolador). De esta manera se pretende contrastar los datos enviados desde el módulo de sensores, con la información recibida por el controlador y la plataforma IoT.

El análisis de desempeño del controlador se realiza contrastando los parámetros de referencia $r(t)$, con los datos de realimentación $h(t)$ por medio de gráficas en el tiempo obtenidas de los datos almacenados.

La evaluación de la comunicación inalámbrica por radio frecuencia se realiza mediante el software XCTU de Digi internacional [27], a través del cual se mide la recepción de datos a diferentes distancias y la intensidad de señal RSSI de las siglas en inglés Received Signal Strength Indication [14].

Por otro lado, se realiza medición de consumo de energético tanto en los módulos de sensores como en el controlador, tanto en las funciones de recepción y transmisión de datos, así como en modo reposo.

\section{RESULTADOS Y DISCUSIÓN}

En la Tabla IV se resumen los datos almacenados en modo local y en la nube durante el mes de abril del 2020 de las variables del suelo y ambiente registradas; se evidencia un contraste en el número de registros, esta diferencia está asociada al promedio de las variables medidas que realiza la plataforma IoT, sin embargo, a través de esta se logró realizar el análisis de las variables $h(t)$, aunque, no de las acciones de control $u(t)$ de estado ON/OFF, dado que $u(t)$ oscila durante el tiempo de muestreo. En relación con el almacenamiento local, se obtuvo un promedio $\overline{\mathrm{x}}=77.25(\overline{\mathrm{x}}=$ media aritmética de los registros) registros por hora, infiriendo que valor aproximado de 17 cambios de $u(t)$ en la misma ventana de tiempo, dado que los 60 registros restantes corresponden al almacenamiento por minuto de $h(t)$.

TABLA IV

REGISTRO DE DATOS ALMACENADOS LOCALMENTE Y EN LA PLATAFORMA IOT

\begin{tabular}{|l|c|c|c|c|}
\hline $\begin{array}{l}\text { Datos } \\
\text { guardados en }\end{array}$ & $\begin{array}{l}\text { Total } \\
\text { registros }\end{array}$ & $\begin{array}{l}\text { Registros } \\
\text { / día } \\
\text { promedio }\end{array}$ & $\begin{array}{l}\text { Registros } \\
\text { / hora } \\
\text { promedio }\end{array}$ & $\begin{array}{l}\text { Registros } \\
\text { /minuto } \\
\text { promedio }\end{array}$ \\
\hline $\begin{array}{l}\text { Local- } \\
\text { MicroSD }\end{array}$ & 55620 & 1854 & 77.25 & 1.3 \\
\hline $\begin{array}{l}\text { Nube- } \\
\text { Plataforma IoT }\end{array}$ & 43200 & 1440 & 60 & 1 \\
\hline
\end{tabular}

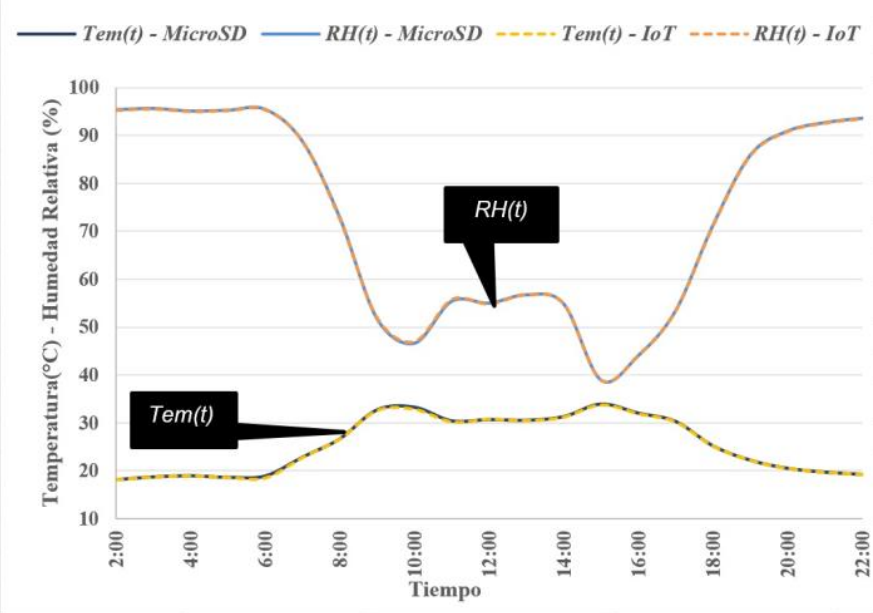

Fig. 6. Contraste de $T e m(t)$ y $R H(t)$ en métodos de almacenamiento implementados 
En la Fig. 6 se observa el comportamiento de $\operatorname{Tem}(t)$ y $R H \%(t)$ (valores medidos por minutos la variable $R H \%(t)$ hace referencia a los valor de $R H(t)$ en porcentaje) en ambos métodos de almacenamiento, obteniendo como resultado una diferencia de $\pm 0.2^{\circ} \mathrm{C}$ en $\operatorname{Tem}(t)$ y $\pm 0.4 \%$ en $R H \%(t)$, este error, puede estar asociado a desconexiones de la plataforma IoT o el controlador; no obstante, a través del gráfico se evidencia un comportamiento en el tiempo de las variables bajo estudio muy similar, capturando la dinámica del fenómeno que se mide.

Por otro lado, la Fig. 7 se ilustra el comportamiento de $\operatorname{Tem}(t)$ y $u(t)$ sobre los ventiladores y extractores de aire en el invernadero. Se observa como la acción de $u(t)$ cambia de estado de acuerdo al valor de referencia de $\operatorname{Tem}(t)$. Por ejemplo, nótese a través de las etiquetas en la gráfica, como a las 10:09 $\mathrm{Tem}(t)$ supera el valor de referencia con $34^{\circ} \mathrm{C}$ y al instante se produce el cambio de estado en $u(t)$ encendiendo los actuadores, y cómo a las 10:11 se apagan los actuadores dado que $\operatorname{Tem}(t)$ es $<34^{\circ} \mathrm{C}$.

Otro punto a considerar es el comportamiento de $u(t)$ cuando esta por fuera de los demás parámetro de referencia $r(t)$, en este caso la hora de encendido y apagado esta 10:00 y 16:00 respectivamente, es importante notar que, siendo las 9:55 y $\operatorname{Tem}(t)>34^{\circ} \mathrm{C}$ no se originó el cambio de estado sobre los actuadores, es decir que la acción $u(t)$ no solo está asociada a las variables $h(t)$ sino que también depende de todos los parámetros de referencia $r(t)$ vistos en el apartado II-A. Algo semejante ocurre cuando los parámetros de referencia $r(t)$ se configuran para control de fertirrigación, donde adicional a las variables $H(t), p H(t)$ y $C E(t)$, también se asocian parámetros de rango y frecuencia en el tiempo, por lo cual esta estrategia de riego de múltiples parámetros de referencia $r(t)$ garantiza que la fertirrigación o control de variables en el un invernadero se puedan ajustar a la necesidad específica de un cultivo.

De manera general, se obtuvo una adecuada acción $u(t)$ en el valor de referencia de $34^{\circ} \mathrm{C}$ dentro de la hora de encendido y apagado establecida en los parámetros de control de temperatura (10:00-16:00). Considerando que en el invernadero se presentaban algunas restricciones de funcionamiento, se aclara que no se realiza el control de $R H \%(t)$.

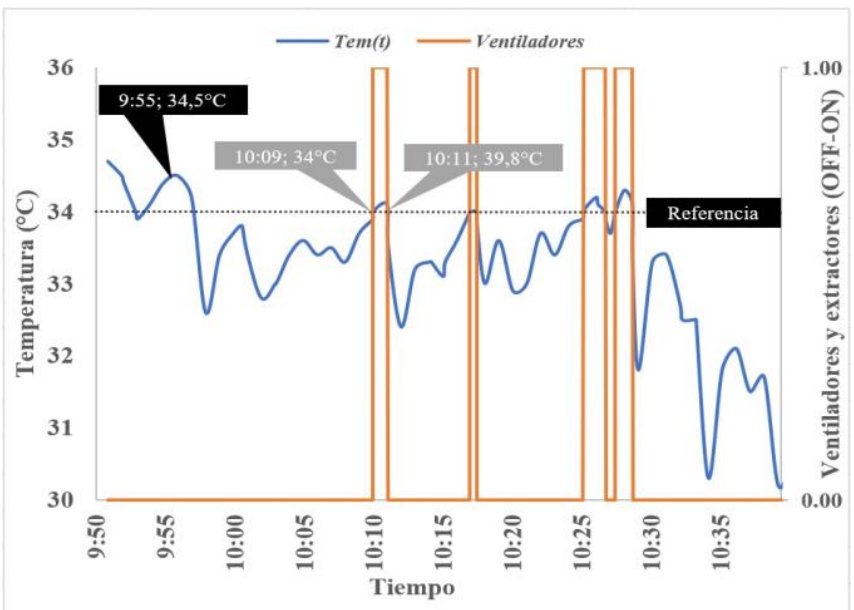

Fig. 7. Comportamiento de la acción de control $u(t)$ sobre Tem(t).
En relación al control de las variables asociadas al suelo, $H(t), p H(t)$ y $C E(t)$, se exponen los resultados en el tiempo en la Fig. 8, contrastando principalmente el comportamiento de la tensión del suelo $H(t)$ con la Tem(t), nótese como el número de riegos diarios varía, este se identifica con un cambio evidente en la variable $H(t)$, claramente el cambio está asociado a la acción $u(t)$ sobre $H(t)$ una vez la tensión supera el valor de referencia de $20 \mathrm{Kpa}$. En síntesis, durante el día de mayor temperatura, el número de riegos fue mayor con 13 riegos en comparación a el día más frío con 7 riegos, logrando así, mantener la tensión en el suelo cercana al valor de referencia de $20 \mathrm{Kpa}$, alcanzando independencia del consumo de agua de la planta o la evapotranspiración.

Por otro lado, la dosificación de nutrientes y solución reguladora de $\mathrm{pH}$ asociadas a las variables $C E(t)$ y $\mathrm{pH}(t)$, se realiza en pequeñas dosificaciones en cada riego, ya que el valor de $C E(t)$ y $p H(t)$ en cada instante de riego se encontraban mínimamente por debajo del valor de referencia, es de notar que se produjo un cambio mínimo de las variables en cada riego, lo que permitió mantener los valores cercanos a la referencia de $p H(t)=6.5$ con y $C E(t)=1.1 \mathrm{~ms} / \mathrm{cm}$.

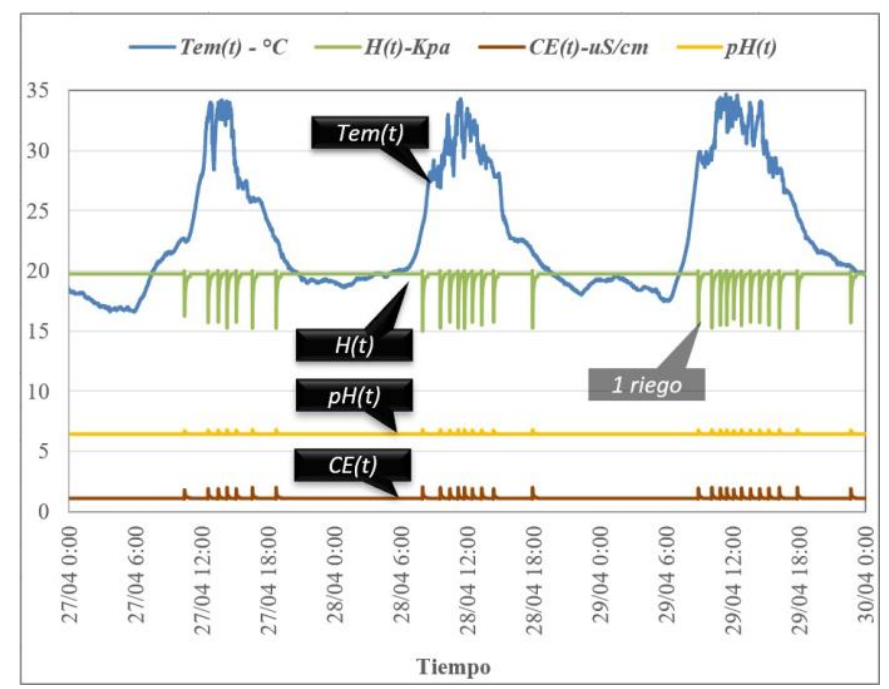

Fig. 8. Comportamiento de las variables en el suelo y temperatura

Por otro lado, en Tabla V se presenta una comparación de los valores de referencia y la capacidad del controlador para preservar estos, se observa el valor de referencia, promedio, mínimo, máximo y error absoluto asociado a cada variable bajo estudio. En relación a $H(t), p H(t)$ y $C E(t)$, el error absoluto fue calculado con respecto a la media aritmética de estos, a diferencia de $\operatorname{Tem}(t)$, donde el error se calculó con respecto a valor máximo de temperatura entre las 10:00 16:00, siendo Tem $(t)$ el error absoluto más alto en $h(t)$; para corregir este, se podría experimentar con ventiladores y extractores de aire de mayor potencia y/o la nebulización de agua en el interior del invernadero, dado que el desvío es derivado de la incapacidad de los actuadores para el control de $h(t)$ y no de la acción $u(t)$ en sí misma. Es importante señalar que estos ajustes no implican una digresión a nivel metodológico, dado que el procedimiento de validación y 
funcionamiento del equipo no se altera por cambios a nivel tecnológico.

TABLA V

RESULTADO DE LA ACCIÓN DE CONTROL

\begin{tabular}{|c|c|c|c|c|c|}
\hline \multirow{2}{*}{$h(t)$} & $\begin{array}{c}\text { Valor de } \\
\text { referencia } \\
r(t)\end{array}$ & $\begin{array}{c}\text { Valor } \\
\text { medio }\end{array}$ & Mínimo & Máximo & Error \\
\hline \multirow{3}{*}{$\operatorname{Tem}(t)$} & - & $20.27{ }^{\circ} \mathrm{C}$ & $16.6^{\circ} \mathrm{C}$ & $35.8^{\circ} \mathrm{C}$ & - \\
\cline { 2 - 6 } & $\begin{array}{c}<34{ }^{\circ} \mathrm{C} \\
10: 00- \\
16: 00\end{array}$ & $31.14{ }^{\circ} \mathrm{C}$ & $\begin{array}{c}22.40 \\
{ }^{\circ} \mathrm{C}\end{array}$ & $35.2{ }^{\circ} \mathrm{C}$ & $1.2{ }^{\circ} \mathrm{C}$ \\
\hline$R H \%(t)$ & - & $79.22 \%$ & $35.6 \%$ & $97.3 \%$ & - \\
\hline$H(t)$ & $<20 \mathrm{Kpa}$ & $\begin{array}{c}19.58 \\
\mathrm{Kpa}\end{array}$ & $14 \mathrm{Kpa}$ & $20 \mathrm{Kpa}$ & $\begin{array}{c}0.42 \\
\mathrm{Kpa}\end{array}$ \\
\hline$p H(t)$ & 6.5 & 6.45 & 6.42 & 6.9 & 0.4 \\
\hline$C E(t)$ & $1.1 \mathrm{mS} / \mathrm{cm}$ & $\begin{array}{c}1.13 \\
\mathrm{mS} / \mathrm{cm}\end{array}$ & $\begin{array}{c}1.05 \\
\mathrm{mS} / \mathrm{cm}\end{array}$ & $\begin{array}{c}1.45 \\
\mathrm{mS} / \mathrm{cm}\end{array}$ & $\begin{array}{c}0.35 \\
\mathrm{mS} / \mathrm{cm}\end{array}$ \\
\hline
\end{tabular}

En relación con el alcance de radio frecuencia RF y la eficiencia en la transmisión y recepción de datos de manera inalámbrica entre el módulo inalámbrico coordinador y el nodo de sensores, estos se enseñan en la Tabla VI, tenido como resultado una cobertura total en el invernadero de prueba. Si se analiza el valor RSSI máximo y mínimo, se infiere que el valor de $-40 \mathrm{dBm}$ indica óptima señal y $-77 \mathrm{dBm}$ una señal más débil pero suficiente para la comunicación entre dispositivos. Es importante aclarar que teóricamente el alcance puede llegar en condiciones ideales hasta $15.5 \mathrm{~km}$ en exterior y $610 \mathrm{~m}$ en interiores [14], sin embargo, la distancia máxima lograda para transmitir datos en esta experiencia fue de $150 \mathrm{~m}$.

TABLA VI

PARÁMETROS DE EVALUACIÓN DE LA COMUNICACIÓN INALÁMBRICA

\begin{tabular}{|c|c|c|c|c|c|}
\hline Radio & $\begin{array}{c}\text { Paquetes } \\
\text { enviados }\end{array}$ & $\begin{array}{c}\text { Paquetes } \\
\text { recibidos }\end{array}$ & $\begin{array}{c}\text { RSSI } \\
\text { sensores } \\
\mathbf{d B m}\end{array}$ & $\begin{array}{c}\text { RSSI } \\
\text { coordinador } \\
\mathbf{d B m}\end{array}$ & $\begin{array}{c}\text { Eficiencia } \\
\%\end{array}$ \\
\hline $30 \mathrm{~m}$ & 100 & 100 & -40 & -40 & $100 \%$ \\
\hline $60 \mathrm{~m}$ & 100 & 100 & -47 & -50 & $100 \%$ \\
\hline $100 \mathrm{~m}$ & 100 & 100 & -56 & -57 & $100 \%$ \\
\hline $150 \mathrm{~m}$ & 100 & 100 & -70 & -77 & $100 \%$ \\
\hline
\end{tabular}

Los resultados de las pruebas de consumo energético del controlador y nodo de sensores se muestran en la Tabla VII. Se considera un tiempo para la trasmisión, recepción de datos y estado de reposo del controlador y en módulo de sensores, así obtenemos una media ponderada (MP) de consumo de $620.8 \mathrm{~mA}$ en el controlador y $18.95 \mathrm{~mA}$ en el módulo de sensores.

TABLA VII

CONSUMO ELÉCTRICO

\begin{tabular}{|l|c|c|c|c|}
\hline Estado & $\begin{array}{c}\text { Tiempo } \\
\text { Controlador } \\
\text { Medio }\end{array}$ & $\begin{array}{c}\text { Consumo } \\
\text { Controlador } \\
\text { Medio }\end{array}$ & $\begin{array}{c}\text { Tiempos } \\
\text { módulo de } \\
\text { sensores }\end{array}$ & $\begin{array}{c}\text { Consumo } \\
\text { módulo } \\
\text { Sensores }\end{array}$ \\
\hline $\begin{array}{l}\text { Transmisió } \\
\text { n de datos }\end{array}$ & $2 \mathrm{~s}$ & $1040 \mathrm{~mA}$ & $2 \mathrm{~s}$ & $240 \mathrm{~mA}$ \\
\hline $\begin{array}{l}\text { Recepción } \\
\text { de datos }\end{array}$ & $5 \mathrm{~s}$ & $854 \mathrm{~mA}$ & $2 \mathrm{~s}$ & $54.4 \mathrm{~mA}$ \\
\hline Reposo & $5 \mathrm{~s}$ & $220 \mathrm{~mA}$ & $60 \mathrm{~s}$ & $10.4 \mathrm{~mA}$ \\
\hline & $\begin{array}{c}\text { Valor medio } \\
\text { ponderado }\end{array}$ & $620.8 \mathrm{~mA}$ & $\begin{array}{c}\text { Valor medio } \\
\text { ponderado }\end{array}$ & $18.95 \mathrm{~mA}$ \\
\hline
\end{tabular}

Los resultados ensañados en la tabla VII sugiere que el algoritmo que rige el funcionamiento del controlador y módulo de sensores restringe el consumo energético cuando no existe solicitud de transmisión y recepción de datos, esta estrategia logra entonces menor consumo de potencia activa, este resultado es importante dado que el controlador mantiene las variables en los valores de referencia y al mismo tiempo minimiza el consumo de corriente eléctrica.

\section{V.CONCLUSIONES}

El conjunto de dispositivos como sensores, actuadores, comunicación e interfaz de usuario del controlador funcionaron según lo esperado, consiguiendo sostener las variables en estudio cercanas a un valor de referencia. Adicional, la validación se basó en contrastar datos vinculados a la nube con los recopilados en sitio, de esto se desprenden varios elemento importantes, el primero evidencia que contar con un sistema offline y online es estratégico para la administración del controlador, por ejemplo la interfaz de usuario Offline permite administrar el controlador cuando no hay disponibilidad de conexión a internet, permitiendo la toma de decisiones en sitio, ahora en segundo lugar, la interfaz de usuario online a través de la plataforma IoT es un instrumento que no sólo permite operar en tiempo real el controlador sino que además permite el monitoreo, supervisión, medición y toma decisiones sobre el cultivo únicamente accediendo a red.

Aspectos fundamentales como la comunicación de los periféricos de entrada y salida del controlador, y el consumo eléctrico funcionaron de manera satisfactoria, tal es el caso de la trasmisión y recepción de datos inalámbrica con un desempeño del $100 \%$, y un ahorro significativo de consumo energético al implementar estado de reposo en el controlador y nodo de sensores. Además, la estrategia de control de múltiples parámetros permite realizar un control integral de las variables que tienen incidencia en el desarrollo de un cultivo, es decir que con un único controlador se logra realizar el control de varias variables ambientales y del suelo en un cultivo.

La metodología de validación del prototipo implementada muestra que a partir de la identificación de las variables a controlar y la implementación de la estrategia de control de múltiples parámetros, se logra un control del proceso en valores de referencia fijados por el usuario. No obstante, la metodología se debe fortalecer con el estudio de otras variables importantes en el desarrollo de cultivos, por ejemplo, el oxígeno disuelto disponible para la planta y la Radiación Fotosintéticamente activa PAR [28]. Por lo tanto, el trabajo futuro debe ir dirigido a validar el controlador en relación al desarrollo productivo de uno a o varios cultivos incorporando otras variables donde se contraste con técnica de producción agrícola tradicional y otros desarrollos tecnológicos disponibles en el mercado. Por otro lado, el procesamiento de datos para la implementación de métodos de inteligencia artificial también debe ser abordado [29].

\section{AGRADECIMIENTOS}

A directivos y equipo de trabajo del Centro de Investigación en Agricultura y Biotecnología CIAB de la Universidad Nacional Abierta a Distancia UNAD por apoyar la investigación, así como al Tecnoparque Nodo Pereira por el 
apoyo metodológico y el acceso a equipos e infraestructura del laboratorio.

\section{REFERENCIAS}

[1] Liotta, M. A., Carrión, R. A., Ciancaglini, N., \& Olguin Pringles, A,"Riego por goteo". PROSAP INTA, Bueno aires, 2015.

[2] DANE, "El cultivo del tomate de mesa bajo invernadero, tecnología que ofrece mayor producción, calidad e inocuidad del producto", 2014. Disponible

https://www.dane.gov.co/files/investigaciones/agropecuario/sipsa/in sumos_factores_de_produccion_dic_2014.pdf.

[3] F. Capraro, Santiago Tosetti., "Herramientas modernas de gestión en riego de precisión basadas en dispositivos electrónicos, programas informáticos y técnicas de control automático.", Electron. J. SADIO, vol. 19, n ${ }^{\circ}$ 1, pp. 47-63, mar. 2020. Disponible en: https://www.sadio.org.ar/index.php/EJS/article/view/154.

[4] L. A. Herrera Fonseca, O. D. Garcia Pabon, "Diseño de un sistema de fertirrigación por goteo para la optimización de agua y fertilizantes en cultivos de cebolla larga del municipio de Aquitania (Boyacá)", Universidad Santo Tomás, Boyacá, 2019. Disponible en: http://repository.usta.edu.co/handle/11634/20942.

[5] C. Molanes Miovich y T. L. Córdova Miranda, "Diseño e implementación de un sistema electrónico de control basado en FPGA, para las condiciones climáticas de un invernadero para el cultivo de tomate.”, INGENIERÍA INVESTIGA, Vol 1, num 1. Pag 96-106. dic. 2019. [En línea]. Disponible en: http://revistas.upt.edu.pe/ojs/index.php/ingenieria/article/view/126.

[6] López A.H.A., Vargas, G. A., Ortiz, C. J. D., Vergara, J.D.S.,"Diseño de un sistema de control y automatización de temperatura, humedad del suelo y humedad relativa para optimizar el rendimiento de cultivos bajo cubierta en CORHUILA", 4to Congreso Internacional AmITIC, Popayán Julio 2017.

[7] R. M. de Anda López, R. Aguirre Aranda, H. Jaramillo Plata, F. Betanzos Castillo, y E. Jiménez Campuzano, E. J. CAMAZAMA, "Sistema inteligente de fertirrigación y control ambiental para invernaderos de zonas tropicales, un detonante económico para horticultores y floricultores de la zona sur del Estado de México", II Congreso Iberoaméricano en Admisnistración de Empresas Agropecuarias, Guanaguato, Mayo 2019.

[8] L. A. Coronel Rojas, D. Rico Bautista, F. R. Cuesta Quintero, E. Barrientos Avendaño, y E. A. Pedraza Villadiego, "Software móvil para reconocimiento, seguimiento y control de las plantas del vivero inteligente de la universidad francisco de paula Santander Ocaña.”, Rev. Colomb. Tecnol. Av., vol. 1, $\mathrm{n}^{\circ}$ 33, mar. 2019, doi: 10.24054/16927257.v33.n33.2019.3329.

[9] J. A. Perez Venzor, A. Delgado Salidos, H.M. Loya Caraveo, D. Garcia Chaparro, A. J. Torres Pedraza, "Sistema De Monitoreo Y Control Para Invernadero Usando La Unidad De Adquisición De Datos Agilet 34970a E Interfaz En Labview", Pistas Edicativas, Vol 40 No 132, Junio 2019.

[10] I. V. Haro Vilaña, F. D. Morales Arevalo, "Implementación de un sistema de monitoreo y control automático de riego para invernaderos mediante tecnología LORA con ESP32", Tesis de licenciatura, Quito, 2019,. Disponible en: http://repositorio.uisrael.edu.ec/handle/47000/2142.

[11] F. F. Escamilla Martínez, "Automatización y telecontrol de sistemas de riego", Trabajo de grad, Grandia diciembre 2016, Disponible en: https://riunet.upv.es:443/handle/10251/75908.

[12] Blynk, "Blynk IoT platform", https://docs.blynk.cc/.

[13] Arduino, "Arduino Due Tech Specs", disponible en: https://store.arduino.cc/usa/due.

[14] DIGI, "XBee-PRO 900HP/XSC RF Modules". Digi International, 2019,Disponible:https://www.digi.com/resources/documentation/dig idocs/pdfs/90002173.pdf.

[15] NodeMCU Team, "NodeMCU Documentation", disponible en: https://nodemcu.readthedocs.io/en/master/

[16] Solomon Systech, "Solomon Systech SSD1963 Datasheet". https://www.crystalfontz.com/controllers/SolomonSystech/SSD196 $3 /$.

[17] Xptek Technology co, "XPT2046 Touch Screen Controller Data Sheet", 2046. Disponible en: http://www.xptek.com.cn

[18] Electronics Co, "Digital temperature and humidity sensor
AM2315", Disponible en: https://cdn-
shop.adafruit.com/datasheets/AM2315.pdf. $\begin{array}{lll}\text { shop.adafruit.com/datasheets/AM2315.pdf. } & \\ \text { Irrometer, "Tensiómetro modelo SR", } 2020 .\end{array}$ https://www.irrometer.com/pdf/IRROMETERs/105sp-Model SRWEB.pdf.

[20] HANNA Instruments, "HI 1090B/5 Electrodo combinado de pH", 2020. https://www.hannacolombia.com/productos/producto/hi1090b5-electrodo-combinado-de-ph-conexion-bnc-5-m

[21] HANNA Instruments, "HI 98143 Transmisor de $\mathrm{pH}$ y CE con salida galvánica aislada", 2020. https://www.hannacolombia.com/productos/producto/hi-98143transmisor-de-ph-y-ce-con-salida-galvanica-aislada

[22] HANNA Instruments, "HI 3001 Sonda de Conductividad de flujo continuo",

https://www.hannacolombia.com/productos/producto/hi-3001-

sonda-de-conductividad-de-flujo-continuo.

[23] OMROM, "Introduction SSR stands for Solid State Relay", 2020. Disponible en: https://www.omron.com.tw/data_pdf/mnu/y10801_solid_state_relays.pdf.

[24] UNAD, "CIAB - Centro de Investigación de Agricultura y Biotecnología - Universidad Nacional Abierta y a Distancia UNAD”. https://estudios.unad.edu.co/ciab-centro-de-investigacionde-agricultura-y-biotecnologia.

[25] Á. Castaño, M. Aristizábal, y H. González, "Requerimientos hídricos del plátano dominico- hartón (Musa aab simmonds) en la región de Santagueda", Rev. U.D.C.A Actual. Divulg. Científica, vol. $15, \mathrm{n}^{\mathrm{o}} 2, \quad$ pp. 331-338, 2012. Disponible en: https://doi.org/10.31910/rudca.v15.n2.2012.831

[26] L. F. Mejia Gutierrez, “ Evaluación de comportamiento físico y químico poscosecha del plátano dominico Harton (Musa AAB Simmonds) cultivado en el municipio de Belalcázar (Caldas)", Tesis de maestría, Bogotá 2013. Disponible en: https://repositorio.unal.edu.co/bitstream/handle/unal/11897/0110746 8.2013.pdf? sequence $=1$.

[27] DIGI,"XCTU- Digi International" Software, https://www.digi.com/products/embedded-systems/digi-xbee/digixbee-tools/xctu.

[28] B. Sen Wu, A. S. Rufyikiri, V. Orsat, y M. G. Lefsrud, "Reinterpreting the photosynthetically action radiation (PAR) curve in plants", Plant Scencei., vol. 289, p. 110272, dic. 2019, doi: 10.1016/j.plantsci.2019.110272.

[29] K. Jha, A. Doshi, P. Patel, y M. Shah, "A comprehensive review on automation in agriculture using artificial intelligence", Artificial Intelligence in Agriculture, vol. 2, pp. 1-12, jun. 2019, doi: 10.1016/j.aiia.2019.05.004.

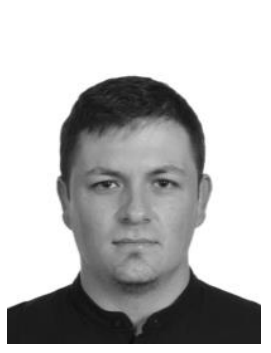

Yenier Valencia Villegas. Ingeniero en Mecatrónica, diciembre 2014, UTP, Pereira Colombia. Especialista en Educación Superior a Distancia, marzo 2018, UNAD, Dosquebradas Colombia. Tecnólogo En Diseño E Integración De Automatismos Mecatrónicos, agosto 2009, SENA, Dosquebradas Colombia. Docente de la Universidad Nacional Abierta y a distancia UNAD e investigador del Centro de Investigación en Agricultura y Biotecnología CIAB de la UNAD. Sus áreas de interés son la agricultura de presión, el internet de las cosas y el desarrollo agroindustrial

ORCID: https://orcid.org/0000-0003-2019-7691

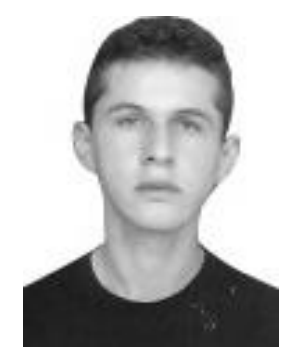

Carlos Alberto Henao Baena: Obtuvo el grado ingeniero electricista de Universidad Tecnológica de Pereira año 2012 y Magister en Ingeniería eléctrica de la misma Universidad en el 2015, fue en el 2013 joven investigador Colciencias, también se ha desempeñado como docente en la facultad de Ingeniería de la Universidad Tecnológica de Pereira, desde el 2016 hasta el 2020 actuó como Gestor de la Línea de Electrónica y Telecomunicaciones del Tecnoparque Nodo Pereira, su áreas de interés son el diseño electrónico, los modelo probabilísticos y el

aprendizaje de máquinas.

ORCID: http://orcid.org/0000-0001-9873-8211 


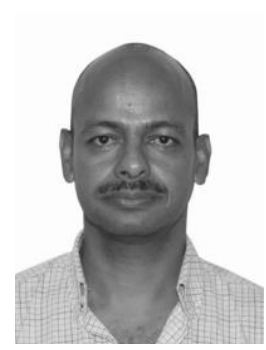

Manuel Francisco Polanco Puerta. Ingeniero Agrónomo. 1994. Universidad Nacional de Colombia Sede Palmira; Especialista Universitario Internacional en Fruticultura, 2000. Universidad Politécnica de Valencia, Valencia España. Magister en Ciencias Agrarias con énfasis en Fitomejoramiento 2011, Universidad Nacional de Colombia Sede Palmira. Doctor en Desarrollo Sostenible: Economía, Sociedad y Medio Ambiente 2016. Universidad Católica Santa Teresa de Jesús de Ávila, España. Docente de la Universidad Nacional Abierta y a Distancia UNAD por más de 17 años, orientando cursos como Fitomejoramiento, Fisiología vegetal, Edafología y fertilidad, Riegos y drenajes. Catedrático de la Universidad de Manizales por tres años como asesor de trabajo de grado de estudiantes de Maestría en Desarrollo sostenible y Medio ambiente y del curso Manejo Integrado del Suelo. Área de interés de investigación: Recursos fitogenéticos, Fitomejoramiento, Desarrollo sostenible; Desarrollo rural; Biotecnología, Fertirrigación, Agricultura de precisión,

ORCID: https://orcid.org/0000-0002-4810-0081 